\title{
Collective phenomena in hadron and nuclear interactions at high multiplicity
}

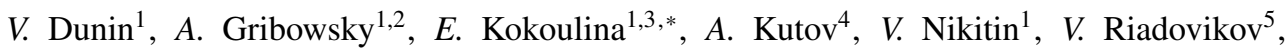 \\ $R$. Shulyakovsky ${ }^{2}, V$. Volkov ${ }^{6}$, and $A$. Vorobiev ${ }^{5}$ \\ ${ }^{1}$ JINR, Joliot-Curie 6, Dubna, Moscow region, 141980, Russian Federation \\ ${ }^{2}$ Institute of Applied Physics, Minsk, Akademicheskaya str. 16, 220072, Belarus \\ ${ }^{3}$ Sukhoi State Technical University of Gomel Prospect Octiabria, 48, 246746, Gomel, Republic of \\ Belarus \\ ${ }^{4}$ Institute of Physics and Mathematics Komi SC UrD RAS, Kommunisticheskaja st., 24, Syk- \\ tyvkar, 167000, Russian Federation \\ ${ }^{5}$ IHEP, Science sq. 1, Protvino, Moscow region, 142281, Russian Federation \\ ${ }^{6}$ Skobeltsyn Institute of Nuclear Physics MSU, Leninskie gory, GSP-1, Moscow 119991, Russian Fed- \\ eration
}

\begin{abstract}
Multiparticle production is described in the language of quarks and gluons. In the experiment the real hadrons are registered. For transfer from quarks and gluons to observed hadrons, various phenomenological models are used. In order to describe the high multiplicity region, we have developed a gluon dominance model. It represents a convolution of two stages. First stage is described by QCD. For second one (hadronisation), the phenomenological model is used. The description of topological cross sections in $p p$ collisions within of our model testifies that in hadron collisions the mechanism of hadronisation is being replaced by the recombination one. At that point, gluons play an active role in the multiparticle production process, and valence quarks are passive. They stay in the leading particles, and only the gluon splitting is responsible for the region of high multiplicity. The model with inclusion of intermediate quark charged topologies describes topological cross sections in $p \bar{p}$ annihilation. We observe the significant growth of a scaled variance of number of neutral pions with increasing of the total multiplicity at U-70 accelerator at IHEP (Protvino). The following experiment with $3.5 \mathrm{~A} \mathrm{GeV}$ deuteron, lithium and carbon beams of the Nuclotron (JINR, Dubna) falling at a carbon target is carried out at the NIS-GIBS setup. The noticeable excess yield of soft photons $\left(p_{T}<50 \mathrm{MeV} / \mathrm{c}\right)$ is observed. The existing models based on Monte Carlo simulation and theoretical estimations predict a lower yield. Our Collaboration plans to study excess of soft photon yield and other phenomena at the SPD (Spin Physics Detector) setup at JINR, Dubna with polarised beams of proton and light nuclei up to $25 \mathrm{GeV}$.
\end{abstract}

\section{Introduction}

Development of high energy physics has been considerably accelerated after the appearance of the strong interaction theory or quantum chromodynamics (QCD) [1]. There were two

\footnotetext{
*e-mail: kokoulina@jinr.ru
} 
experimental stages that influenced its development. The first stage is connected with experiments at hadron accelerators, and the second stage is started with the building of electronpositron colliders. The large volume of the experimental results required the explanation, especially important for description of multiparticle production. Such physicists like Fermi, Feynman, Pomeranchuk, Hagedorn, Dremin, Brodsky and others developed phenomenological schemes. The bootstrap model of Hagedorn predicts the existence of extreme temperature. This behaviour is now interpreted as the formation of quark-gluon plasma.

Using QCD elementary processes, Konishi, Ukava and Veneciano [2] and A. Giovannini [3] built a system of stochastic equations to calculate multiplicity distributions (MD) of partons in quark and gluon jets at high energy collisions. Taking into account the two main elementary processes (gluon splitting and bremsstrahlung of quark with gluon) gives multiplicity distribution (MD) of quarks and gluons in these jets. MD of partons in a quark jet is a well-known negative binomial distribution (NBD), and a Yule-Furry distribution describes MD in a gluon jet.

For comparison with the experimental data, these distributions cannot be used because free quarks are not observed experimentally, due to confinement which has been accepted without proofs so far. To eliminate this difficulty, at the description of MD for $e^{+} e^{-}-$ annihilation to hadrons, a hypothesis of local parton-hadron duality (LoPHD) has been proposed, according to which the hadronization of quarks and gluons occurs softly, without significant momentum transfer between partons. Thus, for description of MD in that process, two stages are taken: the first stage or the quark-gluon ( $q g)$ fission, to which the pQCD can be applied, and the hadronization stage, described phenomenologically. The LoPHD hypothesis was quite consistent with the experiment, while energy of accelerators was not so high to be developing enough $q g$-cascades.

\section{Multiplicity distributions in $e^{+} e^{-}$annihilation}

The $e^{+} e^{-}$annihilation to hadrons is the simplest for analysis, as the produced state is pure $q \bar{q}$. In order to describe this process at energies from several tenths to hundreds $\mathrm{GeV}$, the two-stage model (later renamed the gluon dominance model, GDM) has been offered [4-8]. It's based on the description of MD of partons $P_{m}^{q}$ forming from $q \bar{q}$-pair at the first stage of that process by Giovannini's distributions [3]

$$
P_{0}^{(q)}=\left(\frac{k_{p}}{k_{p}+\bar{m}}\right)^{k_{p}}, P_{m}^{(q)}=\frac{k_{p}\left(k_{p}+1\right) \ldots\left(k_{p}+m-1\right)}{m !}\left(\frac{\bar{m}}{\bar{m}+k_{p}}\right)^{m}\left(\frac{k_{p}}{k_{p}+\bar{m}}\right)^{k_{p}}
$$

with the generating function $(\mathrm{GF}) Q^{(q)}(z)$

$$
Q^{(q)}(z)=\sum_{m=0}^{\infty} z^{m} P_{m}=\left[1+\bar{m} / k_{p}(1-z)\right]^{-k_{p}} .
$$

where $\bar{m}$ determines mean multiplicity of partons and $k_{p}$ is estimated as the ratio of probabilities of two elementary processes, bremsstrahlung and splitting.

We add a phenomenological scheme at the second stage (hadronization of quarks and gluons). In this model, for hadrons from the parton (quark or gluon) jet at the hadronization stage, a binomial distribution (BD) and its GF are

$$
P_{n}^{H}=C_{N_{p}}^{n}\left(\frac{\bar{n}_{p}^{h}}{N_{p}}\right)^{n}\left(1-\frac{\bar{n}_{p}^{h}}{N_{p}}\right)^{N_{p}-n}, \quad Q_{p}^{H}=\left[1+\frac{\bar{n}_{p}^{h}}{N_{p}}(z-1)\right]^{N_{p}}
$$


where $C_{N_{p}}^{n}$ - a binomial coefficient, $\bar{n}_{p}^{h}$ and $N_{p}(p=q, g)$ have meaning of mean multiplicity and maximum number of secondary hadrons what are formed from a single parton on the stage of hadronization.

This choice is based on experimental data [9]. BD has a negative second correlation moment, $f_{2}=\overline{n(n-1)}-\bar{n}^{2}, n$-multiplicity, $\bar{n}$ is its average value, at energies lower $9 \mathrm{GeV}$. In this region the number of partons at the stage of the $q g$-cascade is small, the hadronization is predominant and determines the sign of the second correlation moment.

We suppose that hypothesis of soft decouloration is right. MD of hadrons in $e^{+} e^{-}$annihilation are determined by the convolution of both stages and has a view

$$
P_{n}(s)=\left.\sum_{m=o}^{\infty} P_{m}^{(q)} \frac{1}{n !} \frac{\partial^{n}}{\partial z^{n}}\left(Q^{H}\right)^{2+m}\right|_{z=0},
$$

where $2+m$ is the total number of partons (two quarks and $m$ gluons).

To distinguish hadrons that are created from quarks and gluons we introduce parameter $\alpha=\frac{N_{g}}{N_{q}}$ and simplify designations: $N=N_{q}, \bar{n}^{h}=\bar{n}_{q}^{h}$. After differentiation on $z$ in (4), we obtain MD of hadrons in $e^{+} e^{-}$annihilation ( $M_{g}$ is the maximum number of active gluons)

$$
P_{n}(s)=\sum_{m=0}^{M_{g}} P_{m}^{P} C_{(2+\alpha m) N}^{n}\left(\frac{\bar{n}^{h}}{N}\right)^{n}\left(1-\frac{\bar{n}^{h}}{N}\right)^{(2+\alpha m) N-n} .
$$

We use the term "active" gluon for those gluons what can give gluons of fission with the following quark pair formation. The comparison of (5) with experimental data [10] at 91.4 $\mathrm{GeV}$ is represented on Fig. 1, a. This model describe data from 14 up to $189 \mathrm{GeV}[4,5]$ quite well. The mean gluon multiplicity $\bar{m}$ rises almost logarithmically. The parameter $k_{p}^{-1}$ can be interpreted as temperature $T$, which has tendency to increasing with a growth of energy .

At the second stage, application of the perturbation theory of QCD is difficult. Comparison with experimental data shows the gluon jet is softer, and its parameter $\bar{n}_{p}^{h}$ remains almost constant and close to one in the energy range from 10 to $200 \mathrm{GeV}$ (Fig. 1, b).

Such behaviour confirms the LoPHD hypothesis and the fragmentation mechanism of hadronization [11]. At the fragmentation mechanism, the initial high energy quark emits a bremsstrahlung gluon which splits to $q \bar{q}$ pair. Then a pair's quark picks up a convenient quark (antiquark) from a vacuum and forms an observed meson. In this case, mesons turn out predominant particles. Experiments at RHIC show that the ratio of baryons to mesons is considerably less than one in the peripheral region and it is increasing with the growth of centrality when lots of quarks form dense quark soup. The creation of heavy quark pairs occurs but it is energetically suppressed.

As opposed to the predictions of numerous Monte-Carlo generators, GDM correctly describes the MD on the tail of a large multiplicity, and it also indicates the sources of the observed oscillations of the normalised correlation moments at high energy [6] are namely developed a $q g$-cascade and BD at the hadronization stage.

That scheme of hadronisation, in particular, explains the jump between the average multiplicities in the three-gluon decay of heavy quarkonia $\Upsilon(9.4)$ and $\Upsilon(10.02)$ and in $e^{+} e^{-}$annihilation at the same energy [5]. In this case, GF for the hadron $\Upsilon$-decay is

$$
Q^{(3 g)}(z)=\sum_{m} P_{m}\left[Q^{H}(z)\right]^{m}, P_{m}=\left.\frac{1}{m !} \frac{\partial^{m}}{\partial z^{m}}\left(\frac{z}{\bar{m} / 3}\left[1-z\left(1-\frac{1}{\bar{m} / 3}\right)\right]^{-1}\right)^{3}\right|_{z=0},
$$

where $Q^{H}(z)$ is GF for MD of a gluon jet at the second stage, and MD of gluons in a gluon jet is the Yule-Furry distribution. The average hadron multiplicity is calculated in GDM, and 


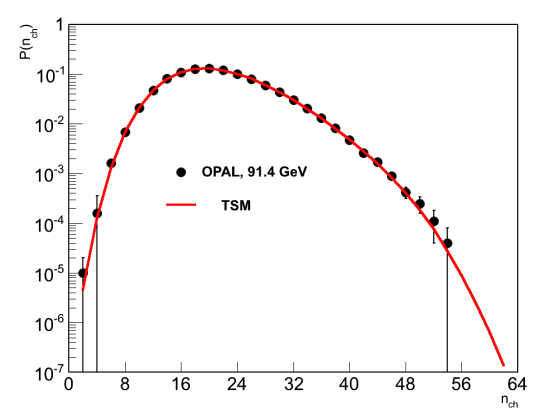

a

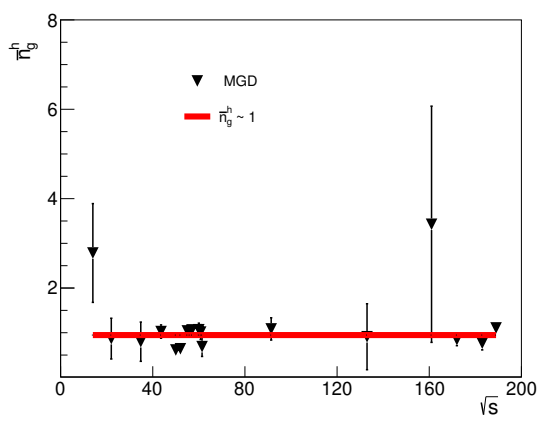

$\mathrm{b}$

Figure 1. a MD hadrons in $e^{+} e^{-}$annihilation at $91.4 \mathrm{GeV}$ [10] is described by red line (5). b The hadronization parameter $\bar{n}_{g}^{h}$ [4] determines the average multiplicity of hadrons formed from single gluon during its passing of the second stage.

it is equal to $\bar{n}^{\Upsilon}=\bar{m} \cdot \bar{n}_{(g)}^{h}$. The average multiplicity in $e^{+} e^{-}$annihilation at the same energy $(\sim 10 \mathrm{GeV})$ in GDM is $\bar{n}(s)=\left(2+\alpha \bar{m}_{(q)}\right) \bar{n}_{q}^{h}$. Their differences, $\Delta \bar{n}=\bar{n}^{\Upsilon}-\bar{n}(s)$, calculated in GDM and experimentally measured coincide.

\section{Proton-proton and proton-antiproton interactions}

The Thermalization project was aimed to search for collective phenomena in $p p$ interactions with a $50 \mathrm{GeV}$ proton beam in the region of multiplicity, which is several times larger than average value. It was advanced in 2004 at JINR (Dubna, Russia). Three institutes carried it out: JINR, IHEP (Protvino) and SINP MSU (Moscow) at the SVD-2 setup (Spectrometer with a Vertex Detector) located on the extracted proton beam of the U-70 accelerator (IHEP).

In this region of multiplicity, such phenomena as the formation of the pion condensate, an increased yield of soft photons, the formation of pion jets of the same sign and others are predicted. Before start of the experiment, Monte-Carlo (MC) simulation of their topological cross sections has been performed. A comparison of it with the data obtained by the Mirabelle collaboration at the U-70 accelerator showed that it significantly (about two orders of magnitude) underestimates the data on the tail of large multiplicity, $n_{c h}=18$. Therefore, we concluded that we should build an improved model consistent with the data in this area.

We guess what hadronization of quarks and gluons in hadron collisions occurs the same way as in $e^{+} e^{-}$annihilation. Just like in $e^{+} e^{-}$annihilation description, our modified GDM takes into account both of stages of multiparticle production. We analysed all available for us data on topological cross sections of hadronic interactions at energies up to several hundred $\mathrm{GeV}$ and found, the existing phenomenological models and $\mathrm{MC}$ codes do not predict right behaviour in the region of high multiplicity. The Mirabelle Collaboration data had indicated to us the direction to develop our model.

We started modelling of a scheme of multiparticle production with the most common case when all valence quarks and a few active gluons (appear at the moment of collision) are participants. After development of the $q g$ cascade new, new formed quark pairs form observable hadrons (mesons and baryons) by combinatorial permutations at hadronization, which is described by BD.

First, we took the scheme with taking into account splitting of active gluons at the first stage. MD for quark and gluon jets are described by NBD and Yule-Furry distributions [3], 
accordingly and BD (5) for hadronization. Based on the hypothesis of soft decoloration for quarks and gluons, we build MD and GF for $p p$ interactions

$$
P_{n}=\sum_{m=0} P_{m}^{P} P_{n}^{H}(m), Q(z)=\sum_{m=0} P_{m}^{P}\left(Q^{H}(z)\right)^{m}=Q^{P}\left(Q^{H}(z)\right)
$$

In this case, parameters, which have the meaning of the average number of hadrons formed from quark or gluon, at the hadronization stage, $\bar{n}_{q(g)}^{h}$, accept values conciderably less than the same parameters at the description of $e^{+} e^{-}$-annihilation. Therefore, we assumed that not all valence quarks are active, and we consistently reduced their number from three pairs to two, to one and, finally, all of them had been completely excluded and left in the leading particles. The parameter $\bar{n}_{g}^{h}$ began to grow and even slightly exceeded the value, corresponding to $e^{+} e^{-}$-annihilation.

In the scheme with gluon splitting, the expression for MD represents convolution of three distributions: MD of gluons at the moment of impact (Poisson) as analogue of impact parameter, Yule-Furry for gluon splitting and BD for the hadronization stage:

$P_{n}=\sum_{k=0} \frac{e^{-\bar{k}} \bar{k}^{k}}{k !} \sum_{m=k} \frac{1}{\bar{m}^{k}} \frac{(m-1)(m-2) \ldots(m-k+1)}{(k-1) !}\left(1-\frac{1}{\bar{m}}\right)^{m-k} C_{\delta m N}^{n-2}\left(\frac{\bar{n}^{h}}{N}\right)^{n-2}\left(1-\frac{\bar{n}^{h}}{N}\right)^{\delta m N-(n-2)}$.

Parameter $\delta$ qualifies the fraction of active gluons that fragment to real observed hadrons. Comparison to the experimental data [12] testifies, this value is equal to $47 \%$, which agrees with theoretical estimation of A. Mueller [13].

In the second scheme, the gluon splitting is not taken into account. The fragmentation of active gluons into hadrons is described in both scenarios the same way. It is assumed all stages of hadron interaction occurs independently of each other. In this approach, the expression for MD is considerably simplified

$$
P_{n}=\Omega \sum_{m=0}^{M} \frac{e^{-\bar{m}} \bar{m}^{m}}{m !} C_{m N}^{n-2}\left(\frac{\bar{n}^{h}}{N}\right)^{n-2}\left(1-\frac{\bar{n}^{h}}{N}\right)^{m N-(n-2)} \quad(n>2)
$$

$\left(P_{2}^{e l}=e^{-\bar{m}}\right)$. The comparison (9) with experimental data [12] (see Fig. 2, a) leads to the following values of parameters $N=4.24 \pm 0.13, \bar{m}=2.48 \pm 0.20, \bar{n}^{h}=1.63 \pm 0.12$ and the normalization factor $\Omega=2$ with $\chi^{2} /$ ndf $\sim 0.5$. We are constrained in sum (9) $M=6$ (the maximal possible number of gluons at the end of the first stage).

Model descriptions of measured at SVD-2 topological cross sections in the high multiplicity region are shown in Fig. 2, a. The gluon (GDM) and so-called a quark models [14] describe data well, NBD overestimates them. GDM describes well experimental topological cross sections of $p p$ interactions from $50 \mathrm{GeV} / c$ at $\mathrm{U}-70$ up to ISR energies [6]. At that, the hadronization parameter $\bar{n}_{g}^{h}$ grows from 1.5 at $50 \mathrm{GeV} / c$ up to 3.3 at $62.2 \mathrm{GeV}$, ISR. The replacement of the hadronization mechanism at transition from the annihilation of leptons to the hadron and nuclei interactions is perfectly illustrated by B. Muller [11]. The growth of the hadronization parameter is the evidence of the implementation of the recombination mechanism of hadronization. This stage occurs in quark-gluon medium but not in vacuum.

The GDM takes into account the predominance of splitting gluons in processes of multiple production at higher energies as superposition of two contributions. The main one occurs from initial gluons, the second one give two gluons, which appear after splitting initial gluon:

$$
\sigma_{n}=\alpha_{1} \sum_{m_{1}} \frac{e^{-\bar{m}_{1}} \bar{m}_{1}^{m_{1}}}{m_{1} !}\left(\begin{array}{c}
m_{1} N_{g} \\
n-2
\end{array}\right)\left(\frac{\bar{n}_{g}^{h}}{N_{g}}\right)^{n-2}\left(1-\frac{\bar{n}_{g}^{h}}{N_{g}}\right)^{m_{1} N_{g}-n+2}+
$$




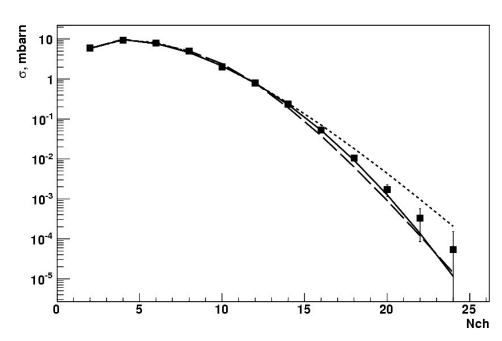

a

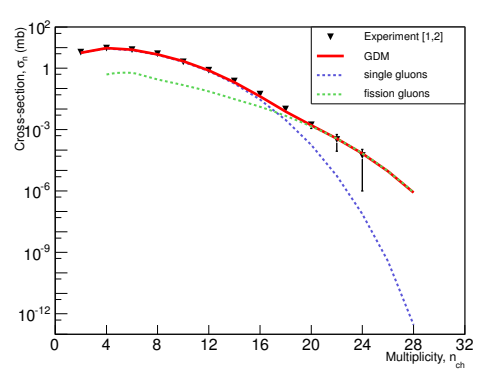

b

Figure 2. (a) Description of topological cross sections, $\sigma\left(N_{c h}\right)$. Comparisons of data $[5,12]$ with the gluon dominance model [5] (solid line), the KNO-based model [14] (long dashed line) and NBD (short dashed line). (b) Topological cross sections $\sigma_{n}$ versus charged multiplicity $n_{c h}$ in the GDM. The dashed blue line describes the contribution of single sources, the green line presents contribution from the sources consisting of two gluons of fission, the solid red line is their sum.

$$
+\alpha_{2} \sum_{m_{2}} \frac{e^{-\bar{m}_{2}} \bar{m}_{2}^{m_{2}}}{m_{2} !}\left(\begin{array}{c}
2 m_{2} N_{g} \\
n-2
\end{array}\right)\left(\frac{\bar{n}_{g}^{h}}{N_{g}}\right)^{n-2}\left(1-\frac{\bar{n}_{g}^{h}}{N_{g}}\right)^{2 m_{2} N_{g}-n+2},
$$

where $m_{1,2}$ are numbers of single and double gluon sources, $\bar{n}_{h}^{h}$ and $N_{g}$ are their parameters of hadronization. In this scheme at the second stage, the branching gluons give twice as large hadrons because they are sources consisting of two gluons (second summand in $\sigma_{n}$ ). The ratio of parameters $\alpha_{1} / \alpha_{2}$ is approximately equal to 1.8 .

In the double-logarithmic approximation [15] it was revealed that the emission of two gluon jets formed by the fission process of a paternal quark (antiquark) is predominant with increasing energy. This fission can give the broadening of topological cross sections at high multiplicity [5, 15]. In Fig. 2,b, the description of measured topological cross sections [5, 12] by GDM (solid red line) is shown. The dashed green line presents the contribution that appeared from the single gluon sources; the dashed blue line (the second summand in (10)) describes the contribution responsible for fission gluons. Obviously, taking into account the gluon fission improves the description of data. It explains discovered in heavy ion collisions at the RHIC setup, and then in proton collisions at LHC in high multiplicity events the longradius correlations called ridges. We have shown [15] the formation of two gluons arising from a bremsstrahlung gluon by splitting at small angles to the initial direction of the valence quark prevails in comparison with the serial emission by that quark of two gluons. In this case, we can observe a very narrow hadron jet with a wide variation of particles in rapidity.

The GDM has been modified for describing the topological cross section of protonantiproton annihilation by introducing intermediate charged quark topologies. This modification is based on well-known experimental fact of leading of two charged pions and an active role of gluons in multiparticle production of hadrons according to GDM. One can form the following combinations from quarks $(u, u, d)$ of the proton and antiquarks $(\bar{u}, \bar{u}, \bar{d})$ of the antiproton: "0"-, "2"-, "4"- and "6"- topologies. Neutral "0"-topology corresponds to the formation of three neutral pions from these partons, "2"-topology - the formation of two charged pions, $\pi^{+}$and $\pi^{-}$, and one neutral, $\pi^{0}$, by using only valence quarks. The remaining "4"- and "6"-topologies form secondary pions not only from valence, but also from quarks of vacuum (sea quarks). Experiments indicate to the leading of two charged pions, which 
confirms this scheme [9]. This scheme describes well the topological cross sections $\Delta \sigma_{n}$ of the pure $p \bar{p}$ annihilation process, which are defined as differences $\Delta \sigma_{n}=\sigma_{n}(p \bar{p})-\sigma_{n}(p p)$ [5].

In the modified GDM, we neglect the rarest "6"-topology. The contribution of the other three combinations can be estimated from the comparison with the data. We find the parameters of hadronization are in agreement with the parameters determined from data of $p p$ interactions with minor deviations. The derived relation between the topologies of " 0 " : "2" $: " 4 "=15: 40: 0.05$ indicates what the main contribution is made by two main combinations of valence quarks ("0" and "2"). As shown in Fig. 3, a, GDM describes differences of topological cross sections between $p \bar{p}$ annihilation and $p p$ scattering at the same energy of colliding hadrons, including the appearance of two local maxima at the field of formation of two and six charged secondary particles. Moreover, "4"-topology is responsible for the appearance of the tail of high multiplicity.

At lower energies, the hadronization stage is predominant. In this case, calculated in GDM, the second correlation moment $f_{2}$ gives negative values and demonstrates the linear growth in the region of negative values with energy. In accordance with GDM, that behaviour is stipulated by increasing the average multiplicity of active gluons in a wide enough energy range in contrast to the diffraction process like $p p$ collisions, in which valence quarks are staying in the leading nucleons. With growth of energy, the contribution of splitting gluons goes up which leads to a sign change of $f_{2}$ from negative to positive and to the broadening of MD.

This modifying scheme of $p \bar{p}$ annihilation can explain behaviour of the second correlative moment. We suggest active gluons emergence together with the formation of intermediate topology occurs. The GF for a single active gluon $Q_{1}(z)=[1+\bar{n} / N(z-1)]^{N}$ gives [5]

$$
f_{2}=\left.Q_{1}^{\prime \prime}(z)\right|_{z=1}-\left[\left.Q_{1}(z)\right|_{z=1}\right]^{2}=-\left(\bar{n}^{h}\right)^{2} / N<0 .
$$

and for $\mathrm{m}$ gluons GF and $f_{2}$ will be

$$
Q_{m}(z)=\left[1+\bar{n}^{h} / N(z-1)\right]^{m N}, \quad f_{2}=-m\left(\bar{n}^{h}\right)^{2} / N .
$$

We consider that $m$ grows while increasing the energy of the colliding particles, and $f_{2}$ will decrease almost linearly as function $m$. Such behaviour qualitatively agrees with experimental data [9]. If we take particular MD $P_{m}^{g}$ for gluons, then GF for secondary hadrons and $f_{2}$ are

$$
\begin{gathered}
Q(z)=\sum_{m} P_{m}^{g}\left[1+\bar{n}^{h} / N(z-1)\right]^{m N} \\
f_{2}=\left[f_{2}^{g}+1-1 / N\right] \cdot \bar{m} \cdot\left(\bar{n}^{h}\right)^{2}, \quad f_{2}^{g}=\overline{m(m-1)}-\bar{m}^{2},
\end{gathered}
$$

In this case, $f_{2}$ may change sign. We consider that the negative value $f_{2}$ at the same energy in comparison with $p p$ interactions can be related with the destruction of the initial system on three fractions with the bigger number of active gluons than in $p p$ collisions.

\section{Pion (Bose-Einstein) condensate}

The transition to study neutral pion production was the next step. We assumed the universality of hadronization in hadron interactions and used the available data from bubble chamber Mirabelle [12] on cross sections of $\pi^{0}$-mesons to describe MD of neutral pions by GDM. It turned out that such parameters of hadronization as the average value and the maximum possible number of $\pi^{0}$-mesons, which are formed at the stage of hadronization from one 
gluon source (active gluon), are comparable with the corresponding values for charged particles. Moreover, in GDM, the ratio between the average multiplicities of charged and neutral mesons is close to $2: 1$ what corresponds to the theoretical value.

Carrying out our experiment at U-70, we restored the multiplicity of neutral pions and investigated the region of high total multiplicity, $n_{t o t}=n_{c h}+n_{0}$, as sum of charged and neutral particles. The SVD-2 setup allowed us to do that, as it includes an electromagnetic calorimeter. This calorimeter registers gamma-quanta. They are decay products of neutral pions, which predominate at U-70 energy. The calorimeter is located on the beam line and behind the detectors that register charged particles.

The restoration of the total multiplicity allowed us to study such a collective phenomenon as the formation of pion or Bose-Einstein condensate (BEC). The theoretical substantiation of its observation has been predicted by the Begun and Gorenstein [16, 17]. They studied the fluctuations of the number of neutral pions at a fixed total pion multiplicity in the ideal pion gas statistical model and showed that the search for pion condensate have to be carried out by selecting events with high total multiplicity when the system is in thermal equilibrium but not chemical equilibrium.

At full equilibrium, the chemical potential of a pion system is equal to zero, and at the formation of the pion condensate, it's approaching to the pion mass. Such a state can be obtained by selecting events with a large number of pions, much more than the average multiplicity. The fluctuations of the number of neutral pions in this case are due to the dynamics of multiple production. The sharp growth of a scaled variance $\left(\omega=D / \bar{n}_{0}=\left(\overline{n_{0}^{2}}-\bar{n}_{0}^{2}\right) / \bar{n}_{0}\right)$ of the neutral pion number with an increase in the total pion multiplicity would be a signal for the BEC formation in proton collisions.

We found there is not any growth of the scaled variance calculated for the simulated events in the whole simulation region. The reconstruction of $\pi^{0}$-mesons is based on using the simulation. Event by event method is impossible in our case. Therefore, we worked out a new method for restoration of the number of events with a certain multiplicity of neutral mesons. About one million events were processed and the number of events with given multiplicity of neutral mesons have been obtained. Among rare events with high total multiplicity we observe events with a number of $\pi^{0}$ mesons comparable to the multiplicity of charged particles: in the event with $n_{\text {tot }}=32$ pions is observed $n_{c h}=16$.

Our data are evidence of an increase of the scaled variance in the region of high total multiplicity, which reaches 7 standard deviations from simulated events at $n_{\text {tot }} \sim 30$ [18]. Fig. 3 , b confirms that result. The smallness of systematic errors in comparison with the statistical errors can be justified by the agreement of our data with Mirabella's data at lower multiplicity. Our theoretical estimations based on the formulas of statistical quantum physics indicate that in events with high total multiplicity, the born pions are in the BEC state.

\section{Soft photon yield in nucleus interactions}

Experimental and theoretical studies of direct photon production in hadron and nuclear collisions essentially expand our knowledge about multi-particle production mechanisms. Until now, we do not have total explanation for the experimentally observed excess of soft photon (SP) yield. These photons have low transverse momenta $p_{T}<0.1 \mathrm{GeV} / \mathrm{c}$ and Feynman variable $|\mathrm{x}|<0.01$. In this domain their yield exceeds the theoretical estimates by $3 \div 8$ times (see [19] and references there).

The theoretical models do not give exhaustive explanation of SP anomalous yield. The model $[5,6]$ explains an excessive SP yield by the production of soft gluons in quark-gluon system. These gluons do not have enough energy to fragment into hadrons, so they are picked 


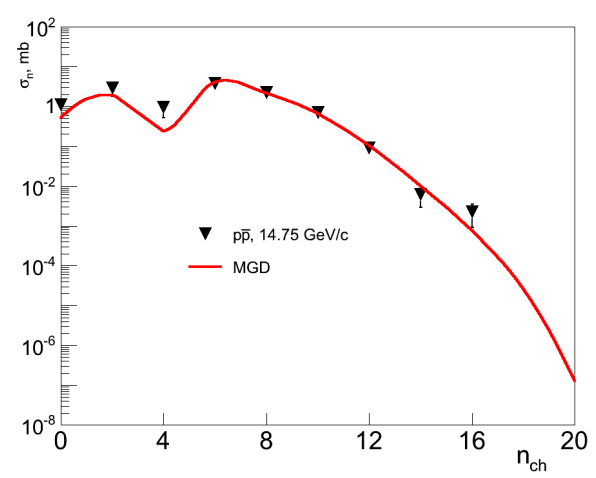

a

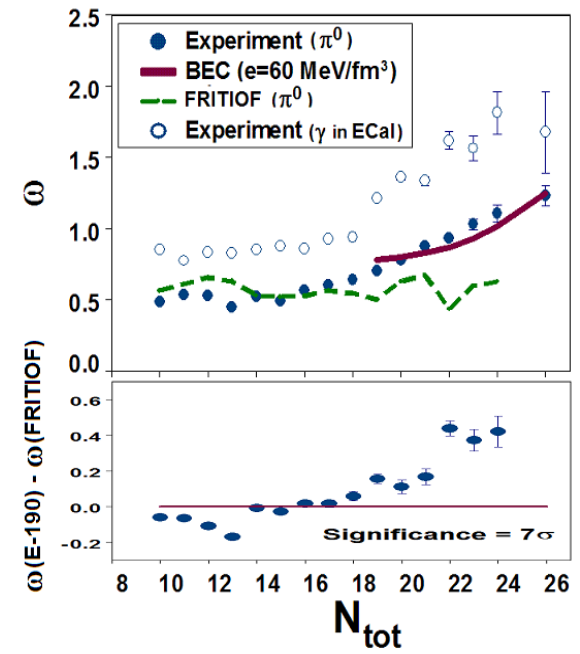

b

Figure 3. (a) Differences of topological cross sections between proton-antiproton and proton-proton $\left(\sigma_{n}=\sigma_{p \bar{p}}-\sigma_{p p}\right)$ interactions at the same energy about ten GeV [5] . (b) (Top) The measured scaled variance $\omega$ versus $N_{\text {tot }}$ for $\pi^{0}$-mesons (•), photons (o), MC code FRITIOF7.02 (the dashed curve) and theoretical prediction (the solid curve) $[16,17]$ for energy density $\epsilon=60 \mathrm{MeV} / \mathrm{fm}^{3} . N_{t o t}=N_{c h}+N_{0}$ for $\pi^{0}$-mesons and $N_{t o t}=N_{c h}+N_{\gamma}$ for photons. (Bottom) The difference of experimental and simulated values of $\omega$.

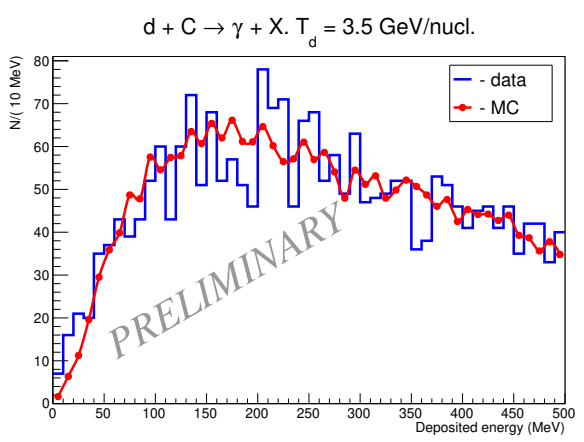

a

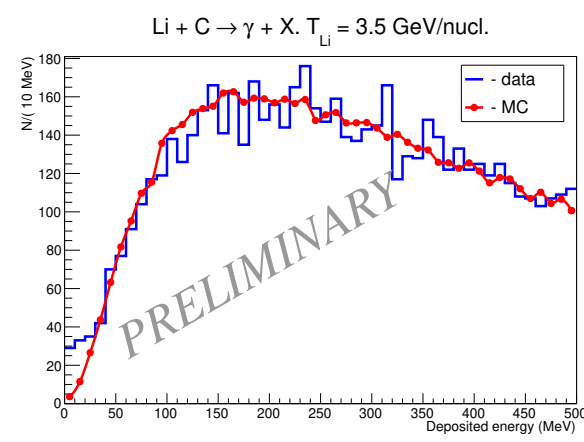

b

Figure 4. a Entire energy spectra in SPEC with pre-shower (a red line) and simulation (a blue line) in $\mathrm{d}+\mathrm{C}$ interactions at $3.5 \mathrm{~A} \mathrm{GeV} / \mathrm{c}$ at Nuclotron. $\mathrm{b}$ The same spectra in $\mathrm{Li}+\mathrm{C}$ interactions.

up the valency quarks of secondary particles and reradiate SP [4]. The parameter $\delta$ determines their fraction (8).

SVD Collaboration manufactured an SP electromagnetic calorimeter (SPEC) on the base of the BGO scintillators (bismuth ortogermanate) [20] to study SP yield at U-70 accelerator and Nuclotron (JINR, Dubna). This calorimeter is a stand-alone device and it differs from many similar ones by its extremely low energy threshold of gamma-quantum registration of order of $2 \mathrm{MeV}$. The SPEC technique permits to execute the unique research program of $p p, p \mathrm{~A}$ and $\mathrm{AA}$ interactions with registration of SP. 
During the Nuclotron run, SPEC was set at an angle of $16^{\circ}$ relative to the beam direction. The front plane of crystals is away from the target at the distance $203 \mathrm{~cm}$. The digitisation of plastic scintillators is realised with a CAMAC ADCs (Lecroy 2249A) and TDCs (LeCroy 2228A), the digitization of analog signals of calorimeter - by ADC CC-008.

MC simulation of the SPEC setup has been carried out at the conditions of the last assembly and the beam energy equal to 3.5 A GeV. URQMD and Geant-3.21 were used. Geant-4 shows the same result.

After data processing we have obtained SP spectra of energy release in deuterium-carbon (Fig. 4, a) and lithium-carbon (Fig. 4, b) interactions. In the region of energy below than 50 $\mathrm{MeV}$, a noticeable excess over MC simulation has been observed. It agrees well to other SP experiments [19].

Our Collaboration plans to make a prolongation of high multiplicity study with direct SP at the future SPD (Spin Physics Detector) setup at JINR, Dubna, which will be established at the building here a new collider NICA. This setup is going to use polarised beams of proton and light nuclei with energy up to $25 \mathrm{GeV}$.

E.K. is grateful to all participants of SVD-2 Collaboration for its active and fruitful work on project.

I appreciate ISMD 2018 organisers for fruitful atmosphere and excellent conditions for discussions.

\section{References}

[1] F. Halzen and A. D. Martin. Quarks and Leptons. Acad. press, NY (1979).

[2] K. Konishi, A. Ukawa and G. Veneziano, Nucl. Phys. B 157, 45 (1979).

[3] A. Giovannini, Nucl. Phys. B 61, 429 (1979).

[4] E. S. Kokoulina, XXXII ISMD, Alushta, Ukraine. 2002. World Scientific. 340 (2002).

[5] E. S. Kokoulina, Phys. Part. Nucl. Lett. 13, 74 (2016).

[6] E. S. Kokoulina, A. Ya. Kutov and L. F. Babichev, Nonlin. Phenom. Complex Syst. 10, 291 (2007).

[7] E. Kokoulina, A. Kutov and V. Nikitin, Braz. J. Phys. 37, 785 (2007).

[8] E. S. Kokoulina, AIP. Conf. Proc. 828, 81 (2006).

[9] J. G. Rushbrooke and B. R. Webber, Phys. Rep. 44, 1 (1978).

[10] P.D.Acton et al. OPAL Collaboration. Z.Physics C 53539 (1992).

[11] B. Muller, Nucl. Phys. A 750, 84-97 (2005).

[12] V. V. Ammosov et al., Phys. Lett. B42, 519 (1972).

[13] A. H. Mueller. Nucl. Phys. A 715, 20 (2003).

[14] S.V. Semenov et al. (In Russian) Yad. Fiz. 22792 (1975).

[15] E. A. Kuraev, S. Bakmaev, and E. S. Kokoulina, Nucl. Phys. B 851, 551 (2011).

[16] V.V. Begun and M.I. Gorenstein. Phys. Lett. B 653 (2007) 190-195.

[17] V.V. Begun and M.I. Gorenstein. Phys. Rev. C 77 (2008) 064903.

[18] E. Kokoulina. Progress of Theoretical Physics. Supplement. 193306 (2012).

[19] J. Abdallah et al. DELPHI Collaboration. Eur. Phys. J. C47, 273 (2006).

[20] V. Dunin et al. Eur. Phys. Journal Web of Conf. 10710005 (2016). 